\title{
Henry Düx Zur Subjekt-Objekt Dialektik in der Rechts- und Staatstheorie der DDR
}

\section{Zur Problemstellung}

Marxistische Rechts- und Staatstheorie haben in der BRD vorwiegend ideologiekritische Funktionen, sofern nämlich der verhüllte Klassencharakter der bürgerlichen Rechtsordnung und der Schein der Neutralität des Staates denunziert werden sollen. Die Schwierigkeiten dieses Unterfangens lassen sich damit kennzeichnen, daß oft genug abstrakt prinzipielle Thesen realanalytische Untersuchungen ersetzen, auf deren Hintergrund aber erst effektive Ideologiekritik und Bausteine einer politischen Strategie möglich wären. Die Funktionsbestimmung einer marxistischen Rechts- und Staatstheorie ist dagegen in der DDR eine andere. Recht und Staat sollen hier die Realität der sozialistischen Gesellschaft gestalten. Beide werden zu einem zentralen Faktor der Absicherung und Weiterentwicklung des Sozialismus. Mit dieser Aufgabenbestimmung werden aber viel komplexere Problemkreise angesprochen, als es auf den ersten Blick scheinen mag. Recht und Staat, die gemäß der marxistischen Theorie dem Uberbaubereich zuzurechnen sind, sollen die gesellschaftliche Basis mitgestalten. Wie verhält es sich mit der materialistischen Grunderkenntnis, daß das Sein das Bewußtsein bestimmt und nicht umgekehrt? Es geht dabei nicht lediglich um die allgemein philosophische Thematik Materialismus, Idealismus, Dialektik, sondern auch theoretisch präziser um die Verhältnisbestimmung yon Basis und Uberbau, um Subjekt-Objekt Dialektik, um das Verhältnis ökonomischer Formbestimmtheiten und Denkformen, um die wissenschaftslogische Dimension des Marxismus, nämlich kategorial logischer Geltung und historischer Genesis. Die scheinbar unwichtigen, weil langsam absterbenden Bereiche des Staats und des Rechts ${ }^{1}$ werden in der DDR - wo Sozialismus qua Anspruch praktiziert wird - zu Aufgaben, die unter dem Vollzugszwang gesellschaftlicher Praxis stehen, zu Fragen, die gelöst werden müssen. Die notwendige praktische Lösung rechts- und staatstheoretischer Fragen $z$ wingt zur Stellungnahme zu den oben genannten komplexen Themenbereichen, die allesamt wesentliche Grundlagen des Marxismus ausmachen. Kritik am Revisionismus "sozialer Staaten " hätte an diesem Punkt anzusetzen, da er Nahtstelle grundlegender theoretischer Einschätzungen und praktischer Po-

\footnotetext{
1 Exemplarisch: Eugen Paschukanis, Allgemeine Rechtslehre und Marxismus, 2. Aufl. Ffm. 1969, insbes. S. I ro ff. Paschukanis' Version des geschichtsteleologisch verlaufenden Absterbeprozesses des Rechts und des Staats wird durch die Realität vieler sozialistischer Staaten widerlegt. Die gegenteilige Entwidklung ist hier zu beobachten. Der Absterbeprozeß kann sich offenbar nur in einem gesellschaftlich geplanten Akt vollziehen, was allerdings die genauesten Kenntnisse der Existenzbedingungen von Recht und Staat voraussetzt, wobei jeder spontaneistische Glaube an einen automatischen Untergangsprozeß ideologisch die entgegengesetzte Wirkung haben muß. Anders und richtig daher: P. I. Studka, Die revolutionäre Rolle von Recht und Staat, Ffm. I969, wo eine Funktionsbestimmung versucht wird. Hier werden allerdings nicht die Bedingungen für einen gesellschaftlich organisierten Absterbeprozeß von Recht und Staat angegeber, so daß gegenteilig die Gefahr der naturnotwendigen Existenz von Recht und Staat heraufbeschworen wird.
} 
litik ist. Drängen in der DDR diese theoretischen Fragen zur praktischen Lösung, so werden die gleichen Themen in der BRD vorwiegend theoretisch unverbindlich diskutiert. ${ }^{2}$ Weder werden solche Theorieansätze zur praktisch folgenreichen Revisionismuskritik genutzt, noch wird die Analyse der Realität der DDR zur Erarbeitung und Fortentwicklung marxistischer Theorie, selbst auf negativer Basis, verwandt. ${ }^{3}$ Die antikommunistische Tradition der BRD hat offenbar selbst in der marxistischen Diskussion ihre Früchte getragen. Von der herkömmlichen Ostrechtsforschung, auch von der im Zuge der Zeit modernisierten ${ }^{4}$, konnte man auf dieser Ebene nichts erwarten, denn mehr als die ideologische Funktion der Legitimation bestehender gesellschaftlicher Verhältnisse besitzt sie nicht.

Die Darstellung marxistischer Rechts- und Staatstheorie in der DDR ist in dieser Arbeit nicht vom Interesse geleitet, einen allgemein informierenden Uberblick über die Diskussionen in der DDR zu liefern, sondern verfolgt im wesentlichen zwei andere Absichten: Erstens soll gezeigt werden, daß marxistische Philosophie kein Terrain abstrakter Spekulation darstellt, daß marxistische Erkenntnistheorie und Wissenschaftslogik von eminent praktischer Bedeutung sind und zweitens soll die Darstellung rechts- und staatstheoretischer Probleme in der DDR ein Problembewußtsein für die Schwierigkeiten beim Aufbau des Sozialismus fördern, soll heißen, die ungeheur sublimen Einflüsse bürgerlicher Theorie und Ideologie auf sozialistische Politik zu analysieren. Zu diesem $Z$ weck werden zwei sich widersprechende und bis vor kurzem konkurrierende Theorieansätze ausgewählt, da sie symptomatische Extrempole in der DDR darstellen. Es soll versucht werden, diese Theorien einer systematischen Kritik zu unterziehen.

\section{Die gesellschaftsorganisierende Funktion des Rechts nach Mollnau}

In der DDR spricht man von der »wachsenden Bedeutung des subjektiven Faktors « und meint die Zunahme der führenden Rolle der Partei, die Zunahme sozialistischer Bewußtheit, denn die Menschen gehen im Sozialismus »nicht mehr von ihrem Willen unabhängige Produktionsverhältnisse ein, sondern bewußte, von ihrem Willen abhängige. ${ }^{5}$ Diese Zunahme des subjektiven Faktors wird in der philosophischen ${ }^{6}$ und ökonomischen ${ }^{7}$ Dimension diskutiert, ohne daß sich

2 Vgl, etwa Alfred Schmidt, Der Begriff der Natur in der Lehre von Karl Marx; G. Markus, J. Zeleny, E. W. Iljenkow, H. G. Backhaus, H. Lefebvre, A. Schmidt in: Beirräge zur marxistischen Erkenntnistheorie Ffm. I969; H. Reichelt, Zur logischen Struktur des Kapitalbegriffs bei Karl Marx, Ffm. 1970; J. Zeleny, Die Wissenschaftslogik und das Kapital, Ffm. 1968; H. J. Krahl, Zur Wesenslogik der Marxschen Warenanalyse, in: Konstitution und Klassenkampf, Ffm. x971, S. 3 I ff.

- Eine Ausnahme bildet: $\Lambda$. Sohn-Rethel, Geistige und körperliche Arbeit, Ffm. 1970, insbes. S. $170-182$

- So besonders Norbert Reich: Marxistische Rechtstheorie zwischen Revolution und Stalinismus in $\mathrm{KJ} \mathrm{72,} \mathrm{I54} \mathrm{ff.;} \mathrm{derselb.} \mathrm{Einleitung} \mathrm{zu} \mathrm{Studka} \mathrm{a.} \mathrm{a.} \mathrm{O.} \mathrm{S.} 7$ ff., dessen positivistische Darstellung historischer Fakten zur marxistischen Rechts- und Staatstheorie ihm genügend Platz lassen, das Material vor dem Hintergrund nicht hinterfragter bürgerlicher Ideologien zu systematisieren und zu bewerten.

5 W. Ulbricht, Freiheit, Wissenschaft und Sozialismus, Berlin 1959, S. 103 f.

- Etwa: H. Taut, Bewußtheit, Spontaneität und Emotionalität im Sozialismus, in: Deutsche Zeitschrift für Philosophie, 1966, Sonderheft S. 7 ff.; G. Klaus, Die Macht des Wortes, Berlin 1964, S. $187 \mathrm{ff}$.

7 Etwa: G. Ebert/F. Matho/ H. Milke, Zu System -und Wirkungsmechanismus der ökonomischen Gesetze im Sozialismus in: Wirtschaftswissenschaft, 1966, S. 529 ff.; A. Dippe/ K. Hartmann: Die wissenschaftliche Führungstätigkeit und der Wirkungsmechanismus der ökonomischen Gesetze im Sozialismus in: Wirtschaftswissenschaft, 1967, S. r ff. 
bislang einheitliche Auffassungen herauskristallisiert hätten. Für K. A. Mollnau, einen der führenden Rechtstheoretiker in der DDR, hat die Zunahme des subjektiven Faktors logische Auswirkungen auf eine ständige Zunahme der Bedeutung des Rechts. ${ }^{8}$ Er sieht sich einig mit den Leitsätzen des Parteiprogramms der SED: "Vordringlich sind die Rechtsnormen zu vervollkommnen, die die wirtschaftlich-organisatorische und kulturell-erzieherische Tätigkeit der Staats- und Wirtschaftsorgane und die Beziehungen zwischen ihnen regeln und zur freien Entfaltung der Kräfte, Talente und Fähigkeiten der Menschen beitragen. « ${ }^{9}$ Mollnau geht davon aus, daß die wachsende Bedeutung des subjektiven Faktors und des Rechts Auswirkungen auf die theoretische Präzisierung bestimmter Bezugsprobleme von Philosophie, Rechtstheorie und politischer Okonomie habe. Wesentlich sind zunächst die Begründungsinstanzen Mollnaus für die Zunahme des subjektiven Faktors und damit implizit die zunehmende Rolle des Rechts im Sozialismus. Auf der allgemein politisch-ökonomischen Ebene sind nach Mollnau »die ökonomischen Verhältnisse gesellschaftliche Erscheinungen, die in allen sozialökonomischen Formationen eine Einheit aus Objektivem und Subjektivem bilden. Beim Entstehen sozialistischer ökonomischer Verhältnisse nimmt indessen der Anteil des Subjektiven qualitativ zu. Die sozialistischen Produktionsverhältnisse entstehen nicht, auch nicht keimhaft, im Schoße des Kapitalismus, sondern sie werden durch die bewußte Tätigkeit der Arbeiterklasse und ihrer Verbündeten unter Führung der Partei hergestellt. Während die kapitalistischen Produktionsverhältnisse in ihrer Gesamtheit naturwüchsig entstehen, wird die Gesamtheit der sozialistischen Produlitionsverhältnisse nach einem vorher entworfenen ideellen Modell aufgebaut. Das System der gesellschaftlichen Verhältnisse des Sozialismus wird in diesem Sinne vom gesellschaftlichen Bewußtsein erzeugt. Wesentliche Bedeutung kommt dabei dem funktionellen Wirken des sozialistischen Rechts zu. «10 Die Behauptung, daß sozialistische Produktionsverhältnisse nicht, auch nicht keimhaft, im Schoße des Kapitalismus entstehen, führt dazu, die Revolution und den Aufbau des Sozialismus zum alleinigen Akt gesellschaftlicher Bewußtheit zu machen, obwohl Marx in der Kritik des Gothaer Programms eindeutig erklärt: "Womit wir es zu tun haben, ist eine kommunistische Gesellschaft, nicht wie sie sich auf ihrer eigenen Grundlage entwickelt hat, sondern umgekehrt, wie sie eben aus der kapitalistischen Gesellschaft hervorgeht, also in jeder Beziehung, ökonomisch, sittlich, geistig, noch behaftet ist mit den Muttermalen der alten Gesellschaft, aus deren Schoß sie herkommt. «11 Entweder leugnet Mollnau diese Ansicht Marxens, die wohl zu den Grundaxiomen des dialektischen Materialismus zählt, oder er geht davon aus, daß die DDR sich bereits in einer Phase klassenloser Gesellschaft befindet; dann aber ist es inkonse-

8 Mit diesem Thema beschäftigt sich Mollnau an verschiedenen Stellen: Bericht von D. Joseph/ G. Udke über eine Sitzung des wissenschaftichen Rats der Juristischen Fakultät der Humboldt-Universität zu Berlin $\rightarrow$ Die Ausnutzung ökonomischer Gesetze mit Hilfe rechtlicher Mitcel im neuen ökonomischen System der Planung und Leitung der Volkswirtschat* in: Staat und Recht I965, S. I00 ff.; ders.: Probleme des sozialistischen Rechtsbegriffs im Lichte der Subjekt-Objekt Dialektik, Beitrag zum Jenenser rechtstheoretıschen Symposium, Mai I966, in: Wissenschaftl. Zeitschrift der Friedrich Schiller Universität Jenas, Gesellschafts- und Sprachwissenschaftl. Reihe I966, S. 423 ff.; Theoretische Probleme der gesellschaftsorganisierenden Funktion des sozialistischen Rechts-Diskussionsbeitrag zum sozialistischen Recht in der Subjekt-Objekt Dialektik, in: Staat u. Recht $1967,715 \mathrm{ff}$.; Staats- und Rechtstheorie, Grundfragen der Theorie des sozialistischen Rechts, Humboldt Universität Berlin Abt. Fernstudium, Heft s, Berlin 1966.

9 Programm der SED Berlin 1963, S. 358.

$10 \mathrm{~K}$. A. Mollnau, Theoretische Probleme der gesellschaftsorganisierenden Funktion des sozialistischen Rechts in: Staat und Recht 1967, 715 ff. (717/718).

11 K. Marx, Kritik des Gothaer Programms, MEW I9, S. 20 Berlin 1972. 
quent, wenn er noch von der Existenz von Recht und Staat ausgeht, denn dieser Institutionen bedarf es bekanntlich dann nicht mehr. Der Staat in der Form der Diktatur des Proletariats und das Recht in der Form des sozialistischen Rechts ist gerade Ausdruck der Tatsache, daß auch im Sozialismus noch Grundelemente der bürgerlichen Gesellschaft vorhanden sind. Der Staat und insbesondere die Diktatur des Proletariats sind nur notwendig, wenn es noch Klassen gibt ${ }^{12}$, das Recht ist nur notwendig, wenn noch materielle Ungleichheiten existieren. ${ }^{13} \mathrm{Nur}$ weil Marx auch im Sozialismus noch von Klassen und Ungleichheiten ausging, konnte er sagen: "Dieser Sozialismus ist die Permanenzerklärung der Revolution, die Klassendiktatur des Proletariats als notwendiger Durchgangspunkt zur Abschaffung der Klassenunterschiede überhaupt, zur Abschaffung sämtlicher Produktionsverhältnisse worauf sie beruhen, zur Abschaffung sämtlicher gesellschaftlicher Beziehungen, die diesen Produktionsverhältnissen entsprechen, zur Umwälzung sämtlicher Ideen, die aus diesen gesellschaftlichen Beziehungen hervorgehen. $\ll^{14}$

Vereint Marx die historische Entfaltung der sozialistischen Produktivkräfte aus den kapitalistischen und die sozialistische Bewußtheit zur Diktatur des Proletariats, so zieht Mollnau diese beiden Komplexe wieder auseinander und kommt zur idealistischen Funktionsbestimmung des Rechts als ideell entworfenem Modell. "Indem das sozialistische Recht auf das Handeln der Menschen wirkt, ihnen Handlungsweisen vorschreibt(!), organisiert, gestaltet, schafft es sozialistische Gesellschaftsverhältnisse, die ihrerseits das Produkt des bewußten wechselseitigen Handelns der (!) Menschen sind. «15 Mit dieser Funktionsbestimmung des Rechts werden allerdings die zentralen Probleme insgesamt ausgespart: was sind die erkenntnistheoretischen Grundlagen der ideellen Modelle, des sozialistischen Rechts, was ist der Inhalt dieses Rechts, wie stellt sich die Genesis der Rechtsform dar, wie kann man von der bewußten Tätigkeit des Menschen schlechthin sprechen, wenn es noch Klassen und Klassenkampf im Sozialismus gibt, d. h. wer entwirft die ideellen Modelle, die rechtstheoretische Elite im Symposium mit anderen Wissenschaftseliten oder die revolutionäre Arbeiterklasse?

Mollnaus Erklärung der wachsenden Bedeutung des Rechts reduziert diese Problemstellung: Das sozialistische Recht stelle auf ökonomischem Gebiet ein konstituierendes Moment der Produktionsverhältnisse dar. Dieser Prozeß werde beschleunigt und vertieft dadurch, daß im Zuge der technischen Revolution die Wissenschaft immer mehr zur unmittelbaren Produktivkraft wird, ein Vorgang, der zu wesentlichen Veränderungen in der Dialektik von Materiellem und Ideellem, von Subjekt und Objekt führt. "Die wachsende Rolle des Rechts bei der Lenkung und Leitung der OOkonomie ist Ausdruck der Stellung bestimmter Bereiche der Rechtswissenschaft im Verwandlungsprozeß der Wissenschaft zur unmittelbaren Produktivkraft. Durch die technische Revolution, die rasch fortschreitende Arbeitsteilung, wird die Produktion derart kompliziert (!), daß zu ihrer Organisation in ständig steigendem Maße wissenschaftliche Erkenntnisse vonnöten sind. Diese Organisation von Produkten ... entsteht nicht von selbst, sie muß geschaffen werden. Der Rechtswissenschaft kommt hier eine große Aufgabe zu, da bestimmte Beziehungen unter den Bedingungen des Sozialismus nur rechtlich or-

$12 \mathrm{~K}$. Marx, Die Klassenkämpfe in Frankreich, MEW 7, S. 88 ff.; Berlin I960 u. Kritik des Gothaer Programms, a. a. O., S. 27 ff.

${ }^{13} \mathrm{~K}$. Marx, Kritik des Gothaer Programms, a. a. O., S. 2 I $>$ Es ist daher ein Recht der Ungleichheit, seinem Inhalt nach, wie alles Recht $\propto$.

${ }^{14}$ K. Marx, Klassenkämpfe in Frankreich a.a.O., S. 89/90.

15 Mollnau, a. a. O., S. 7168 . 
ganisierbar sind und des Rechts bedürfen, wenn sie funktionieren sollen. Die Rechtswissenschaft wird insoweit ... im Prozeß der technischen Revolution zur unmittelbaren Produktivkraft, weil sie sich mit diesen produktionsorganisatorischen Beziehungen befaßt und der Praxis entsprechende Empfehlungen vermittelt. «16 Wenn man wie Mollnau die sozialistische Revolution und den Aufbau des Sozialismus zur ausschließlichen Frage gesellschaftlichen Bewußtseins macht, so muß sich diese Betrachtungsweise in der Reflexion über ökonomische Prozesse fortsetzen. Die wissenschaftlich-technische Revolution gerinnt zum wertneutralen ökonomischen Phänomen, ein Phänomen übrigens, das sich unterschiedslos in imperialistischen und in "sozialistischen " Staaten beobachten läßt. Mollnau erklärt die zunehmende Bedeutung des sozialistischen Rechts gerade mit der wissenschaftlich-technischen Revolution und identifiziert den Prozeß der sozialistischen Revolution im ökonomischen Bereich mit der technischen Revolution. Vollzieht sich diese technische Revolution aber ebenso in imperialistischen Staaten, so sieht Mollnau den Unterschied dennoch darin, daß kapitalistische Produktionsverhältnisse naturwüchsig und sozialistische Produktionsverhältnisse nach ideellen Modellen entstehen. Eine marxistische Interpretation des imperialistischen Staates hat es aber heute damit zu tun, daß man sich auch im Kapitalismus längst ideeller Modelle bei der staatsinterventionistischen Tätigkeit zur Regelung ökonomischer und sozialer Probleme bedient. Für Mollnau wird offenbar die qualitative Seite revolutionärer Veränderung zur lediglich quantitativen Frage. Der Obergang vom Kapitalismus zum Sozialismus wird zu einem in Stadien verlaufenden Zunahmeprozeß gesellschaftlicher Bewußtheit bei ökonomisch im wesentlichen gleichbleibender Situation. Bei einer solchen Betrachtungsweise kann es für Mollnau nicht einmal zum Problem werden, daß unter den Bedingungen der wissenschaftlich-technischen Revolution und selbst der Abschaffung des Privateigentums an den Produktionsmitteln ökonomische Formbestimmtheiten wie Warenproduktion - als Residuen der kapitalistischen Okonomie - auch im Sozialismus noch existentiell sind. Wenn es diese Residuen im Sozialismus nicht mehr gäbe, wären allerdings Recht und Staat überflüssig. Die historisch verfrühte Abschaffung des Geldes etwa in Kuba oder Marxens Kritik an der ArbeitsgeldLehre utopischer Sozialisten weisen gerade auf die Problematik hin, daß die Existenz kapitalistischer ökonomischer Formbestimmtheiten nicht mit dem voluntaristischen Akt sozialistischer Bewußtheit zu überwinden ist. ${ }^{17}$

Für Mollnau scheinen im Gegenteil ökonomische Probleme lediglich technische Produktions-, Zirkulations- und Distributionsfragen zu sein, also Regelungen zur Aufrechterhaltung des Status quo mit historischer Verlängerung. Antagonistische Widersprüche in den Produktionsverhältnissen und auch in der gesamten Gesellschaft gibt es nicht mehr, denn nur dann kann Mollnau vom bewußten Handeln der Menschen und der gesamten Gesellschaft sprechen. Das ist die Version eines Staates des ganzen Volkes und einer Partei des ganzen Volkes. Gesellschaftliche Widersprüche brauchen hier nicht mehr durch bewußte Prozesse gelöst zu werden, im Gegenteil, sie werden hinter den Gittern solchermaßen handfester Ideologien eingemauert. ${ }^{18}$ Weiter ist Mollnaus Behauptung wesentlich,

18 a. a. $\mathrm{O}$

17 Versuche einer OKonomieinterpretation, die die historische Genesis außer acht lassen, stehen der Ricardianischen Auffassung der Okonomie nahe (Linksricardianer), vgl. dazu H. G. Badkhaus, Zur Dialektik der Wertform, in: Beiträge zur marxistischen Erkenntnistheorie, a. a. O., S. $128 \mathrm{ff}$.

18 Bekanntlich stellt diese Frage eine der wesentlichen Kritikpunkte der KP Chinas an der KPdSU dar, Uber den Pseudokomunismus Chruschtschows und die historische Lehre für die 
die sozialistische Produktion würde derart kompliziert, daß immer mehr wissenschaftliche Erkenntnisse notwendig seien. Auch die wissenschaftliche Erkenntnis erscheint hier phänomenologisch neutral, als einfach notwendig. Daß die Entwicklung der Denkform kein im Raum schwebender Prozeß ist, sondern nur auf dem Hintergrund der historischen Genesis der Scheidung in Hand- und Kopfarbeit begreifbar wird und vor allem auflösbar ist, scheint für Mollnau unwichtig zu sein. Mollnaus Herleitung wissenschaftlicher Notwendigkeiten gereicht der trivialen Erkenntnis zur Ehre, daß es so und so arbeitende Menschen naturnotwendig geben müsse, eben Hand- und Kopfarbeiter. Was sich hier in Mollnaus Kopf zur Ideologie versteinert hat, ist seit Kants philosophischer Spekulation über dieses Thema unantastbares Prinzip der bürgerlichen Gesellschaft. Kants Kritik der reinen Vernunt mit der Konzeption der zweifellos existenten Urteile a priori als von Sinneswahrnehmungen unabhängige Verstandespotenz interpretiert, begründet die Autonomie des geistigen Wesens, damit die Existenznotwendigkeit der eigenständigen, getrennt von der Handarbeit, sich vollziehenden Geistesarbeit. Wenn Kant sich fragte, wie denn überhaupt solche Urteile a priori möglich seien und sie mit der "ursprünglich - synthetische Einheit der Apperzeption " behauptet und in der Existenz "transzendentaler Spontaneität" zu beweisen suchte, so scheint dies für Mollnau eine zufriedenstellende Antwort zu sein, obwohl er wissen müßte, daß es keine Antwort ist. ${ }^{19}$ Die Ausklammerung dieses Themas ist für Mollnau folgenreich. Indem nicht nach dem Entwicklungsprozeß juristischer Denkformen gefragt wird, also auch der komplexe Zusammenhang zur Genesis der Warenform entproblematisiert wird, kann der Untergang der juristischen Denkform nicht mehr thematisiert werden, sondern es muß ganz im Gegenteil von der immerwährenden Notwendigkeit des Rechts und der Rechtsformen ausgegangen und sogar von der wachsenden Bedeutung des Rechts gesprochen werden.

Für Mollnau nun hat die technische Revolution rechtstheoretische Konsequenzen. Es muß nämlich das Wechselverhältnis zwischen Basis und juristischem Oberbau neu untersucht werden, das Recht kann nicht mehr ausschließlich unter dem Uberbauaspekt diskutiert werden, wobei er keineswegs eine Verbindung zwischen der Entwicklung der Produktivkräfte und dem juristischen Uberbau leugnet. ${ }^{20}$ Mollnau stößt mit solchen Gedanken in der DDR auf eine Rechtstheorie, die, wie er selbst betont, solche Fragen bislang kaum erkannt hat oder ganz einfach nicht anerkennt. Mollnaus Angriffsziel wird offensichtlich, wenn er formuliert: "Es kommt darauf an, jede mechanisch-materialistische Kausalitätsauffassung aus der Rechtstheorie zu verbannen. ${ }^{21}$ Recht ist danach nicht nur Wirkung ökonomischer Prozesse, sondern ebenfalls Ursache für ökonomische und gesellschaftliche Entwicklung, entfaltet also eine gesellschaftsorganisierende Funktion. ${ }^{22}$ Soweit Mollnau sich gegen das mechanische Moment in der marxistischen Rechtstheorie wendet, ist ihm zuzustimmen, denn wenn Recht lediglich als Reflex ökonomischer Verhältnisse begriffen wird, so hat dies nichts mit dialektischem Materialismus weder im Marxschen Sinn noch im Sinne der Leninschen Abbildtheorie zu tun. Die Geschichte wäre danach ein zäh dahin fließender Strom, auf deren Verlauf Menschen niemals Einfluß nehmen können, gesellschaftlicher Fort-

Welt, Die Polemik über die Generallinie der internationalen kommunistischen Bewegung 1964 , Berlin 1971 , S. 465 ff.

19 Vgl. dazu A. Sohn-Rethel, Geistige und körperliche Arbeit, S. 44 u. 46.

20 Mollnau, a. a. O., S. 719.

21 a. a. O., S. 721 .

22 a. a. O., $721 / 22$. 
schritt oder gar Revolutionen wären undenkbar. Die im Austauschprozeß der Waren hervorgebrachten Ideologien kapitalistischer Warenproduktion, Freiheit und Gleichheit, (soweit Basis-Uberbauverhältnis) besitzen selbstredend Rückwirkungseffekte auf die Basis, sonst wäre etwa der Übergang vom Feudalismus zum Kapitalismus als gesellschaftlich fortschreitende Entwicklung gar nicht möglich gewesen und gleichfalls konstituierten Freiheit, Gleichheit und Eigentum als Axiome des bürgerlichen Rechtssystems überhaupt die Möglichkeit der existentiellen Ungleichheit in der kapitalistischen Warenproduktion, die Mehrwertproduktion ("freier " Lohnarbeiter). Engels schrieb I 890 an Bloch: »Nach materialistischer Geschichtsauffassung ist das in letzter Instanz bestimmende Moment in der Geschichte die Produktion und Reproduktion des wirklichen Lebens. Mehr hat weder Marx noch ich je behauptet. Wenn nun irgendjemand das dahin verdreht, das ökonomische Moment sei das einzig bestimmende, so verwandelt er jenen Satz in eine nichtssagende, abstrakte Phrase. Die ökonomische Basis ist die Basis, aber die verschiedenen Momente des Uberbaus - politische Form des Klassenkampfes und seine Resultate - Verfassungen, nach gewonnener Schlacht durch die siegreiche Klasse festgestellt und Rechrsformen und nur gar die Reflexe aller dieser irdischen Kämpfe im Gehirn der Beteiligten, politische, juristische, philosophische Theorien ... üben auch ihre Einwirkung auf den Verlauf der geschichtlichen Kämpfe aus und bestimmen in vielen Fällen vorwiegend deren Form" ${ }^{23}$. Engels beschreibt aber an dieser Stelle auch nicht mehr, als daß es einen bestimmenden Prozeß gibt, andererseits aber auch einen gegenteiligen. Mollnau führt zur Stützung seiner Ansichten andere Zitate der Klassiker an, die aber ebenfalls nicht über diese allgemeinen Ebenen hinausgehen. Für Mollnau reicht dies jedoch bereits hin, um Schlußfolgerungen zu ziehen, obwohl ganz offensichtlich hier vorab eine wesentliche Aufgabe zu leisten wäre, nämlich das Gesamtwerk von Marx und Engels im Hinblick auf die Verhältnisbestimmungen von Basis und Uberbau, von Materie und Bewußtsein und von Erkenntnissubjekt und -objekt einer systematischen Analyse zu unterziehen, da dies bei Marx und Engels an keiner Stelle konzentriert zu finden ist. Der inhaltliche $Z$ weck dieser Arbeit bestände darin, in der Grenzziehung zwischen dialektischem Materialismus und bürgerlicher Philosophie über die Ebene positivistischer Subsumtion historisch empirischer Fakten unter logische Kategorien hinauszugelangen. Die Schwierigkeit dürfte darin liegen, zu erfassen, welche und vor allem wie logische Kategorien verändert werden müssen, um der Realität adäquat zu sein. Das Aussparen dieser Fragen rächt sich bei Mollnau. Im Gegensatz zum mechanischen Materialismus will er einfach den "dialektischen Determinismus « in der Rechtswissenschaft entsprechend anwenden. ${ }^{24}$ Was bedeutet dialektischer Determinismus in der Rechrswissenschaft?

Mollnau erklärr: "Die Anerkennung des sozialistischen Rechts als Ursache für die Existenz bestimmter gesellschaftlicher Verhältnisse, ihrer Reproduktion und ihrer Entwicklung ist keine Konzession an Thesen des bürgerlichen Rechtsdenkens, die von einem idealistisch verkehrten Verhältnis von Recht und Gesellschaft ausgehen. Die in Rede stehende Ursache-Wirkung-Relation mit dem Recht als Ursache berührt nicht die materielle Determiniertheit des Rechts und hebt dessen ideologischen und Willenscharakter nicht auf. «25 Recht in der Wirkungsposition stellt den Determinismus dar und weil Recht zugleich Ursache sein kann, spricht

23 F. Engels an J. Bloch in: Ausgewählte Briefe, Ost-Berlin 1953, S. s02, so aber nicht nur der walte Engels: vgl. auch Deutsche Ideologie, MEW 3, S. 38.

24 Mollnau, a. a. O., S. 721.

25 Mollnau, a. a. O., $721 / 22$. 
Mollnau von Dialektik. Diese Erklärung kommt ihrerseits keinesfalls über eine einerseits-andererseits Ebene hinaus. Diese Pauschalität ist aber die Ebene, auf der dann die Weiterentwicklung der Gedanken Mollnaus sich vollzieht. So heißt es negativ: "Die Fetischisierung der objektiven Gesetzmäßigkeiten in der Rechtstheorie führt dazu, diese als Schöpfer der sozialistischen Gesellschaftsbeziehungen, namentlich der ökonomischen Verhältnisse vorzustellen, der das "Recht« in seinen Dienst nimmt. Eine solche Rechtsauffassung gräbt eine künstliche Kluft zwischen den Bürgern und dem sozialistischen Recht.« ${ }^{26}$ Was sich hier in der Warnung vor der Fetischisierung objektiver Gesetzmäßigkeiten andeutet, wird später zu einer Behauptung verdichtet: »Das Recht ist aber keine erkenntnistheoretische Kategorie und die Rechtstheorie keine erkenntnistheoretische Disziplin, wohl aber haben beide wichtige erkenntnistheoretische Aspek$t^{27}$." Unter Erkenntnistheorie versteht Mollnau hier das Verhältnis von gesellschaftlichem Sein und Bewußtsein, aus deren Dimensionen er die Rechtstheorie ausklammert, um die Ebene dann unverbunden und abstrakt wieder hineinzuholen; er spricht vom selbstverständlichen Primat der materiellen Lebensverhältnisse gegenüber dem Rechtsbewußtsein und dem Recht - und dem unproblematischen Primat der Basis gegenüber dem juristischen Überbau. Die Unklarheiten über den ganzen Problemkreis führen zum kruden Nebeneinander zweier Dimensionen der Rechtstheorie:

I. einer selbstverständlichen erkenntnistheoretischen Seite, dem Primat des Seins und der Basis und

2. einer Subjekt-Objekt dialektischen Ebene, wobei die Beziehung zwischen dem "praktisch-gegenständlich tätigen Menschen und seiner natürlichen und gesellschaftlichen Umwelt ${ }^{28}$ gemeint ist, also nicht das Verhältnis von Materie und Bewußtsein.

Mollnau bemißt der Subjekt-Objekt Dialektik große Bedeutung bei. Er meint, mit dieser Konstruktion sei »eine rechtstheoretische Prämisse gewonnen, von der aus die schwierige Dialektik erörtert werden kann, die bei der Leitung gesellschaftlicher Prozesse mit Hilfe des sozialistischen Rechts wirkt. «29 Und »das sozialistische Recht auf der Basis der Objekt-Subjekt Relation zu erforschen, bedeutet vor allem, das Recht als Teil des Subjekts zu behandeln..$^{30}$

Die unreflektierte Ubernahme der einerseits existenten Basis-Uberbau Relation und dem andererseits postulierten Rüdkkopplungseffekt führt bei Mollnau zur einseitigen Verabsolutierung der Subjekt-Objekt Dialektik. Die metaphysische Trennung von erkenntnistheoretischen Prozessen (Sein bestimmt das Bewußtsein) und der Subjekt-Objekt dialektischen Ebene (Einwirkung der menschlichen Aktion auf die ökonomische Basis) hat ihren Ursprung bei Mollnau darin, daß er die ökonomische Basis als kontinuierliche, neutrale Daseinsinstanz faßt. Dies wurde besonders daran deutlich, wie er mit der technisch-wissenschaftlichen Revolution theoretisch arbeitet. Für ihn wird die Besonderheit der Subjekt - Objekt Dialektik zum eigenständigen, sich getrennt vollziehenden Prozeß neben und außerhalb der Basis-Oberbau Relation, weil er zwar die Ricardianische politischökonomische Differenzierung von Wesen und Erscheinung (real, aber verborgene Wesentlichkeit der Warenproduktion und ihrer Erscheinung auf der Oberfläche) erkennt, nicht aber mehr das qualitativ neue Moment in der Theorie Marxens.

\footnotetext{
26 a. a.O., S. 723.

27 a. a. O., S. 735 .

28 a. a. O., 726 .

20 a. a. $0 ., 727$

30 a. a $O$.
} 
Marx sah nämlich im Unterschied zu Ricardo das Wesen nicht als ein ewig daseiendes naturnotwendiges Produkt, sondern gerade die Veränderlichkeit des Wesens selbst in der historischen Genese. Nach Marx ist deswegen das Wesen der Produktion von Waren eben nicht wie bei Ricardo die abstrakte Arbeit, sondern die ganze Ware als Zellenform der kapitalistischen Produktion, die immer schon den Widerspruch von Gebrauchswert und Tauschwert als prozessierende Dialektik in sich trägt. Wenn Mollnau allerdings das Wesen zu einer neutralen Instanz erklärt, wird für ihn eine gesonderte, selbständige Subjekt-Objekt Dialektik notwendig, obwohl diese Subjekt-Objekt Dialektik ihr Existenzmedium gerade der Widersprüchlichkeit der ökonomischen Zelle Ware verdankt. Die gesellschaftlich bewußte Aktion ist überhaupt nur deswegen möglich, weil dieser Widerspruch von Gebrauchswert und Tauschwert in der logischen und historischen Fortführung - deren Vermittlungsinstanz die Wertformanalyse ist - an die Oberfläche drängt, und der Erkenntnis und Erfahrung zugänglich ist. ${ }^{31}$

$\mathrm{Da}$ Mollnau diese Problematik außer acht läßt, zieht er die marxistische Rechtstheorie auf die erkenntnistheoretische Ebene Kants zurück, indem er Recht als natürlich notwendiges, gesellschaftliches Phänomen setzt, (die Verhältnisse werden kompliziert, deswegen ist Wissenschaft, insbesondere Rechtswissenschaft als Urteil a priori notwendig) und indem er die Subjekt-Objekt Dialektik aus der Basis-Uberbau Relation simplifizierend herausoperiert. Mollnaus Rechtstheorie ist ein Beispiel dafür, wie aus der richtigen Kritik am mechanischen Materialismus die falsche Konsequenz einer Rechtstheorie Kantscher Provenienz gezogen wird.

III. Die Kritik Mollnaus in der DDR und die entgegengesetzte Position in der Rechts- und Staatstbeorie

Auf dem Hintergrund der Subjekt-Objekt Dialektik versuchte Professor Mollnau zusammen mit Professor Klenner ein offiziöses Lehrbuch der Rechtstheorie der DDR herauszugeben. Auf einer Kollegiumssitzung zu rechtstheoretischen Fragen wurde die Arbeit besonders von den maßgeblichen Rechtstheoretikern der DDR, Professor Weichelt, Vorsitzender des Verfassungs- und Rechtsausschusses der Volkskammer, und Professor Arlt, Rektor der Deutschen Akademie für Staats- und Rechtswissenschaft "Walter Ulbricht" kritisiert, so daß es nicht zur Ausführung dieses Vorhabens kam. ${ }^{32}$ Eher auf einer Behauptungsebene, lief die Kritik wesentlich darauf hinaus, daß die Verfasser von einer Verselbständigungstendenz des Rechts in allen sozialistischen Ländern ausgehen, was gefährlicherweise dazu führe, daß das Recht eine selbständige, von der objektiven Realität getrennte Eigenexistenz erhalte. Die Abhebung der Rechtstheorie von der Staatstheorie verkenne, daß die Diktatur des Proletariats wesensmäßige Grundlage aller sozialistischen rechts- und staatstheoretischen Probleme sei, der Kontinuität des Rechts werde das Wort geredet, und die Frage der Zielfunktionen des sozialistischen Systems werde nicht aufgeworfen. Insgesamt handele es sich um einen Angriff des Klassengegners, der erfahrungsgemäß seine ideologischen Anstrengungen immer wieder auf das Kernstück des Marxismus-Leninis-

31 Vgl. zu diesem Fragenbereich J. Zeleny, Die Wissenschaftslogik und das Kapital, a.a. O., S. $19-184$.

$22 \mathrm{Vgl}$. dazu H. Heichtfuß, Bericht, Kollegiumssitzung zu rechtscheoretischen Fragen, in: Staat und Recht 1969, S. 106 ff. 
mus, auf die Lehre von der Diktatur des Proletariats und damit auf die Staatsund Rechtstheorie der Arbeiterklasse richte.

Ist eine grundlegende Kritik an der Rechtstheorie Mollnaus notwendig, so sagen die wortradikalen Behauptungen jedoch noch nichts aus über die inhaltliche Position seiner Kritiker. Diese Untersuchung soll sich wieder an einem exemplarisch herangezogenen Aufsatz vollziehen, der zwar keine Mollnaukritik enthält, aber eine Staats- und Rechtstheorie auf dem Boden der wesensmäßigen Diktatur des Proletariats versucht zu entfalten und damit einem der Hauptkritikpunkte an Mollnau Rechnung trägt.

J. Becher und P. Friedrich versuchten kürzlich unter dem Titel »Beziehung zwischen Basis und Oberbau «33 einen Beitrag zur gesellschaftsgestaltenden Rolle des sozialistischen Staats zu liefern. ${ }^{34}$ In dem Aufsatz wird davon ausgegangen, daß das Wesen des sozialistischen Staats die Diktatur des Proletariats ist. Der Inhalt der Diktatur des Proletariats bestehe darin, "die gesellschaftliche Entwicklung durch die Arbeiterklasse und ihre marxistisch-leninistische Partei zu führen, die sozialistischen Produktions- und Gesellschaftsverhältnisse planmäßig zu organisieren, die politisch-moralische Einheit des Volkes weiter zu festigen und die sozialistischen Errungenschaften vor feindlichen Anschlägen zu schützen. « ${ }^{35} \mathrm{Die}$ sozialistische Staatsmacht sei somit das wichtigste Instrument und politische Organisationsform zur Errichtung der entwickelten sozialistischen Gesellschaft. Gerade der - im Unterschied zu jeder anderen Staatsmacht - vorhandene schöpferische Charakter des sozialistischen Staats erfordere aber ein grundlegenderes Erfassen der Wechselbeziehungen $z$ wischen sozialistischer Basis und dem Uberbau. Im Sozialismus spiegele der Oberbau nicht nur die Basisverhältnisse wider, sondern ihm komme gegenüber der Basis relativ selbständige Bedeutung zu und er spiele eine eigenständige aktive Rolle in der gesellschaftlichen Entwicklung. Das qualitativ neue Element des sozialistischen Staats wird so formuliert: "In der Verwirklichung seiner ökonomischen Funktionen leitet er den gesellschaftlichen Reproduktionsprozeß im umfassenden Sinne, übt er in Form der Leitung produktive Tätigkeit im Rahmen des gesellschaftlichen Gesamtarbeiters aus. «36 Dies führe zu einer Veränderung des Öberbaus dahingehend, »daß dieser zum Instrument der bewußten Leitung und Lenkung der von sozialen Antagonismen befreiten Gesellschaft wird. « ${ }^{37}$ Dieser Veränderungs- und Erneuerungsprozeß des Uberbaus habe zur Folge, daß man durchaus $»$ die These vom Basischarakter der ökonomischen Funktionen des sozialistischen Staats ${ }^{3{ }}$ ", der selbst Uberbaueiement sei, vertreten könne. Die Auffassung vom sozialistischen Staat als politische Organiastion, die Oberbaucharakter besitzt, zugleich aber einem Staat als Gestaltungsfaktor der sozialistischen Basis im funktionalen Sinne bedeute nicht,

3s J. Becher/P. Friedrich in: Staat und Recht 1972, S. I4Io ff.

34 Ahnlich: S. A. Golunski: Zum Begriff der Rechtsnorm in der Theorie des sozialistischen Rechts in: Stzat und Recht 196r, S. I545 ff.; R. Rost: Die führende Rolle des SED bei der Entwicklung des sozialistischen Staats und Rechts in der DDR in: Staat und Recht 1966, S. 72 I ff.; G. Stiller: Aktuelle Schlußfolgerungen aus der Lehre Lenins für die sozialistische Rechtstheorie in: Staat und Recht 1970, S. 575 fi.

35 Becher/Friedrich, a. a. O., S. I4I 3.

36 a. a. O., S. 1414 .

37 a. a. O., S. 1416 .

38 a. a. O., S. I4I6. Zu dieser These gibt es in der DDR eine breite Diskussion: z. B. U. J. Heuer: Staat, Wirtschaft und Wirtschaftsrecht in: Wirtschaftsrecht 1970, S. 8 ff.; P.-B. Schulz: Zur Dialektik von Basis und Uberbau - ihre Bedeutung für die weltanschauliche Orientierung der Staats- und Rechtspraxis in: Neue Justiz 1970, S. I ff.; W. Loose: Kämpferisch unsere Wcltanschauung propagieren und verwirklichen in: Staat und Recht 1972, S. 363 ff., der als Kritik an dieser These ausführt, daß der Staat dann zu einer Basiskategorie werden müßte. 
daß der Staat Ursache für gesellschaftliche Entwicklung - anders also als Mollnau - sei und auch nicht Basiselement werde. $Z$ war thematisieren Becher und Friedrich die Staats- und Rechtstheorie nicht mehr in einer losgelösten SubjelktObjekt Dialektik und legen auch verbal die Diktatur des Proletariats zugrunde, unterscheiden sich aber doch von Mollnau in der positivistisch-neutralen Betrachtung der ökonomischen Prozesse im Sozialismus.

Nur eine solche Konzeption läßt es zu, von dem Problem noch existenter Warenproduktion, und damit produzierter Widerspruchsformen wie etwa Arbeitsteilung zu abstrahieren. Gerade die Widersprüche von vorhandener Warenproduktion und aufzulösender Warenproduktion, von vorhandener Arbeitsteilung und prozeßhaft aufzulösender Arbeitsteilung, von noch bestehenden antagonistischen Klassenwidersprüchen und in der Entwicklung des Sozialismus aufzuhebender Klassenwidersprüche machen das Wesen und zugleich die Schwierigkeiten der Diktatur des Proletariats aus. Die widerspruchsfreie Betrachtung des ökonomischen Prozesses führt aber gegenteilig zur Leugnung dieser Aufgaben, nämlich vorhandene Warenproduktion und Arbeitsteilung erst gar nicht als Problem zu formulieren und in ideologischer Korrespondenz davon auszugehen, daß soziale Antagonismen bereits beseitigt sind. ${ }^{39}$ Nur diese Begriffslosigkeit gegenüber der ökonomischen Basis kann in der DDR dazu führen, daß die Abschaffung des Privateigentums an den Produktionsmitteln zum verabsolutierten Kern des Systems der sozialistischen Produktionsverhältnisse erklärt wird.40 Das reduzierte Verständnis von der Diktatur des Proletariats setzt sich in der Betrachtung des sozialistischen Staats auf dem Hintergrund der Basis-Uberbau Dialektik fort: "Der sozialistische Staat wird als Repräsentant des gesellschaftlichen Gesamtarbeiters unter diesem Aspekt (gemeint ist die sozialistische Bewußtheit, der Verf.) direkt in die planmäßige Organisation der sozialistischen Produlstion, in den Wirtschaftsmechanismus der sozialistischen Gesellschaft einbezogen. «41 Dies führt dann gemäß der Basis-Öberbau Relation zur Aufsplitterung funktionaler Teilbereiche des sozialistischen Staats, einmal als politische Organisation (Uberbaufaktor) und zum anderen zum ökonomischen Gestaltungsfaktor (hier Staat als Basiskategorie). Es wird zwar apologetisch behauptet, daß dies »keinerlei strukturelle Zuordnung des sozialistischen Staates zur Basis ${ }^{42}$ « bedeute, da nur die ökonomischen Funktionen des Staats Basischarakter hätten. Die Trennung in einen Staat als solchen und in partikulare Staatsfunktionen verrät den idealistischen Charakter der Staatstheorie von Becher und Friedrich. Sie kommen zu einer wertneutralen, positivistischen Funktionsbestimmung des Staates im ökonomischen Bereich aber nur deswegen, weil sie den ökonomischen Prozeß insgesamt von dieser Warte aus betrachten.

Dies sind zugleich die bekannten Grundlagen der Theorie vom staatsmonopolistischen Kapitalismus und der antiimperialistischen Demokratie; eine Auffassung, die von der wertneutralen ökonomischen Formbestimmtheit und von neutralen staatlichen Funktionsbestimmungen ausgeht, bei deren Überführung in den Sozialismus es mit der Abkappung des kapitalistischen Managements und dem Einsatz sozialistisch bewußter Spezialisten einerseits und der Umwandlung eines bürgerlichen Parlaments in ein sozialistisches andererseits getan ist. Daß der kapitalistische Produktionsprozeß die widersprüchliche Einheit von Arbeits-

$39 \mathrm{Vgl}$. Becher/Friedrich, S. 1416 is. 1422.

40 a. a. O., S. 142I, während in der UdSSR immerhin noch konkurrierende Ansidten vertreten werden: etwa Planmäßigkeit oder Kollektıvität und kameradschaftliche Zusammenarbeit.

41 a. a. O., S. 1419

42 a. a. O. 
und Verwertungsprozeß darstellt, erscheint nicht mal mehr als Frage. Auf diesem Boden wird in der DDR das Verhältnis von kapitalistischer Ókonomie und Staat so bestimmt: »In unserer Zeit spielen die Beziehungen zwischen Staat und Wirtschaft eine immer größere Rolle. Es ist eine Tatsache, daß in allen kapitalistischen Iändern, die den Ubergang zum staatsmonopolistischen Kapitalismus vollzogen haben, der moderne bürgerliche Staat im Interesse der großen Monopole immer mehr in den kapitalistischen Reproduktionsprozeß eingreift, auf die Gestaltung und Entwicklung der Wirtschaft »aktiv« Einfluß nimmt. Der kapitalistische Staat reguliert die Wirtschaft. «43 Danach sind also die Probleme, die Imperialismus und Sozialismus haben, die gleichen, nur ist der Staat eben hier "reeller Gesamtkapitalist « und dort »reeller Gesamtarbeiter «. Nur die politische Form ist eine andere, während in der ökonomischen Formbestimmtheit eigentlich alles beim alten bleibt. Und dazu berutt man sich auf Lenin, der lediglich verlangt habe, den Gewaltapparat zu zerschlagen, nicht aber den Apparat, der große Arbeit auf dem Gebiet der Rechnungsführung und Registrierung leistet. »Also die Banken, Post, das Telegrafenwesen, die Genossenschaften dürfen nicht zerschlagen werden, sondern müssen zu einem Apparat des Volkes, müssen in einen sozialistischen Apparat umgewandelt werden. ${ }^{44} \mathrm{Daß}$ es heute um die Beseitigung kapitalistischer Formbestimmtheiten bereits vergesellschafteter Produktivkräfte; bei Lenin aber um die Entfaltung der Produktivkräfte im Stadium »ursprünglicher Akkumulation « ging, gerät hier vollends aus dem Blickfeld. Die Aufteilung und Trennung in eine neutrale ökonomische Potenz und eine unterschiedliche politische Potenz bezogen auf unterschiedliche Stellungen des Staats im Basis-Uberbau Verhältnis kann aber in der DDR höchstens legitimationsideologischen Charakter für die nicht vollzogene Revolutionierung der gesellschaftlichen Verhältnisse haben.

Subjekt-Objekt oder Basis-UTberbau dialektischer Betrachtungen, das zeigt die rechts- und staatstheoretische Diskussion in der DDR, können solange nicht mehr als die Aufrechterhaltung des gegebenen gesellschaftlichen Status quo bedeuten, als die dialektische Betrachtung der Widersprüche auf der ökonomischen Basis selbst ausgeklammert wird. Diese Verkürzung ist aber nicht einseitig den aufgeführten Autoren anzulasten, sondern vielmehr dem allgemeinen ideologischen Konsens der UdSSR-orientierten Staaten, daß es auf der ökonomischen Basis keine Widersprüche mehr gäbe, was zwangläufig zur Leugnung sozialer Widersprüche führen muß. Auf diesem Boden muß allerdings eine marxistische Staatsund Rechtstheorie zur funktionalisierten gesellschaftichen Regelungstheorie, einer Systemtheorie mit marxistisch-leninistischer Nomenklatur denaturieren. ${ }^{45}$

\footnotetext{
43 Ingo Wagner, Studien zur marxistisch-leninistischen Revolutionstheorie, Leipzig 1967, S. 3.

44 a. a. O. S. I4

45 Diese Behauptung soll und kann nicht verschleiern, daß es wesentliche Aufgabe ist, die Unterschiede von bürgerlicher Systemtheorie in der BRD und gesellschaftl. Regelungstheorie in der DDR herauszuarbeiten, da dies Grundlage der Bestimmung des Widerspruchs beider gesellschaftlicher Systeme ist. Zugleich könnte die Analyse gesellschaftlicher Regelungstheorie in der DDR Kriterien herausarbeiten, die notwendig sind zur Differenzierung von unterschiedlichen Phasen des Sozialismus, nämlich noch vorhandener gesellschaftlicher Antagonismen und aufgelöster Antagonismen. Jedenfalls kann die Differenzbestimmung nicht so verlaufen, daß einfach behauptet wird, es gäbe keine Antagonismen mehr.
} 